\title{
Weaving Synchronous Reactions into the Fabric of SSA-form Compilers
}

\author{
HUGO POMPOUGNAC, Inria, France \\ ULYSSE BEAUGNON and ALBERT COHEN, Google, France \\ DUMITRU POTOP BUTUCARU, Inria, France
}

\begin{abstract}
We investigate the programming of reactive systems combining closed-loop control with performanceintensive components such as Machine Learning (ML). Reactive control systems are often safety-critical and associated with real-time execution requirements, a domain of predilection for synchronous programming languages. Extending the high levels of assurance found in reactive control systems to computationally intensive code remains an open issue. We tackle it by unifying concepts and algorithms from synchronous languages with abstractions commonly found in general-purpose and ML compilers. This unification across embedded and high-performance computing enables a high degree of reuse of compiler abstractions and code. We first recall commonalities between dataflow synchronous languages and the static single assignment (SSA) form of general-purpose/ML compilers. We highlight the key mechanisms of synchronous languages that SSA does not cover-denotational concepts such as synchronizing computations with an external time base, cyclic and reactive $\mathrm{I} / \mathrm{O}$, as well as the operational notions of relaxing control flow dominance and the modeling of absent values. We discover that initialization-related static analyses and code generation aspects can be fully decoupled from other aspects of synchronous semantics such as memory management and causality analysis, the latter being covered by existing dominance-based algorithms of SSA-form compilers. We show how the SSA form can be seamlessly extended to enable all SSA-based transformations and optimizations on reactive programs with synchronous concurrency. We derive a compilation flow suitable for both high-performance and reactive aspects of a control application, by embedding the Lustre dataflow synchronous language into the SSA-based MLIR/LLVM compiler infrastructure. This allows the modeling of signal processing and deep neural network inference in the (closed) loop of feedback-directed control systems. With only minor efforts leveraging the MLIR infrastructure, the generated code matches or outperforms state-of-the-art synchronous language compilers on computationally intensive ML applications.
\end{abstract}

CCS Concepts: • Software and its engineering $\rightarrow$ Embedded software; Compilers; Domain specific languages; • Theory of computation $\rightarrow$ Operational semantics;

Additional Key Words and Phrases: MLIR, lustre, SSA, machine learning (ML), reactive programming

\section{ACM Reference format:}

Hugo Pompougnac, Ulysse Beaugnon, Albert Cohen, and Dumitru Potop Butucaru. 2022. Weaving Synchronous Reactions into the Fabric of SSA-form Compilers. ACM Trans. Arch. Code Optim. 19, 2, Article 22 (March 2022), 25 pages.

https://doi.org/10.1145/3506706

Authors' addresses: H. Pompougnac and D. P. Butucaru, Inria, France; emails: \{hugo.pompougnac, dumitru.potop\}@inria.fr; U. Beaugnon and A. Cohen, Google, France; emails: \{ulysse, albertcohen\}@google.com.

Permission to make digital or hard copies of all or part of this work for personal or classroom use is granted without fee provided that copies are not made or distributed for profit or commercial advantage and that copies bear this notice and the full citation on the first page. Copyrights for components of this work owned by others than ACM must be honored. Abstracting with credit is permitted. To copy otherwise, or republish, to post on servers or to redistribute to lists, requires prior specific permission and/or a fee. Request permissions from permissions@acm.org.

(C) 2022 Association for Computing Machinery.

1544-3566/2022/03-ART22 \$15.00

https://doi.org/10.1145/3506706 


\section{INTRODUCTION}

The Static Single Assignment (SSA) form [20, 21] has proven an extremely useful tool in the hands of compiler builders. First introduced as a representation to facilitate optimizations, it became a staple of optimizing compilers. More recently, its semantic properties-e.g., functional determinism while still allowing for limited concurrency-established it as a sound basis for Highperformance-computing (HPC) compilation frameworks such as Multi-level Intermediate Representation (MLIR) [17], where different abstraction levels of the same application ${ }^{1}$ share the structural and semantic principles of SSA, allowing them to co-exist while being subject to common analysis and optimization passes (in addition to specialized ones).

But while compilation frameworks such as MLIR concentrate the existing know-how in Highperformance Computing (HPC) compilation for virtually every execution platform, they lack a key ingredient needed in the high-performance embedded systems of the future-the ability to represent reactive control and real-time aspects of a system. They do not provide first-class representation and reasoning for systems with a cyclic execution model, synchronization with external time references (logical or physical), synchronization with other systems, tasks and I/O with multiple periods and execution modes.

And yet, as we shall see in this article, while the standard SSA form does not cover these aspects, it shares strong structural and semantic ties with one of the main programming models for reactive real-time systems: dataflow synchrony $[3,12]$, and its large and structured corpus of theory and practice of reactive systems design.

Contribution. Relying on this syntactic and semantic proximity, we extend the SSA-based MLIR framework to open it to synchronous reactive programming of real-time applications. We illustrate the expressiveness of our extension through the compilation of the pure dataflow core of the Lustre language. This allows us to model and compile all data processing, computational and reactive control aspects of signal processing and machine learning applications. In the compilation of Lustre, following an initial normalization phase, all data type verifications, buffer synthesis, and causality analysis can be handled using existing MLIR SSA algorithms. Only the initialization analysis specific to the synchronous model (a.k.a. clock calculus or analysis) requires specific handling, leading to significant code reuse.

The MLIR embedding of Lustre is non-trivial. As modularity based on function calls is no longer natural due to the cyclic execution model, we introduce a node instantiation mechanism. We also generalize the usage of the special undefined/absent value in SSA semantics [9] and in low-level intermediate representations such as Low-level Intermediate Representation (LLVM IR) [15]. We clarify its semantics and relate it to the notion of absence and the associated static analyses (clock calculi) of synchronous languages [2].

Our extension remains fully compatible with SSA analysis and code transformation algorithms. It allows giving semantics and an implementation to all correct SSA specifications. It also supports static analyses determining correctness from a synchronous semantics point of view.

Outline. Section 2 formalizes the SSA semantics. Section 3 extends SSA to allow reactive synchronous programming. Section 4 covers the embedding of Lustre into MLIR SSA and its compilation. In Section 5, we present our use cases. We discuss related work in Section 6 before the conclusion in Section 7.

\footnotetext{
${ }^{1}$ Ranging from ML dataflow graphs and linear algebra specifications down to affine loop nests and optimized (tiled, vectorized, etc.) low-level code.
} 


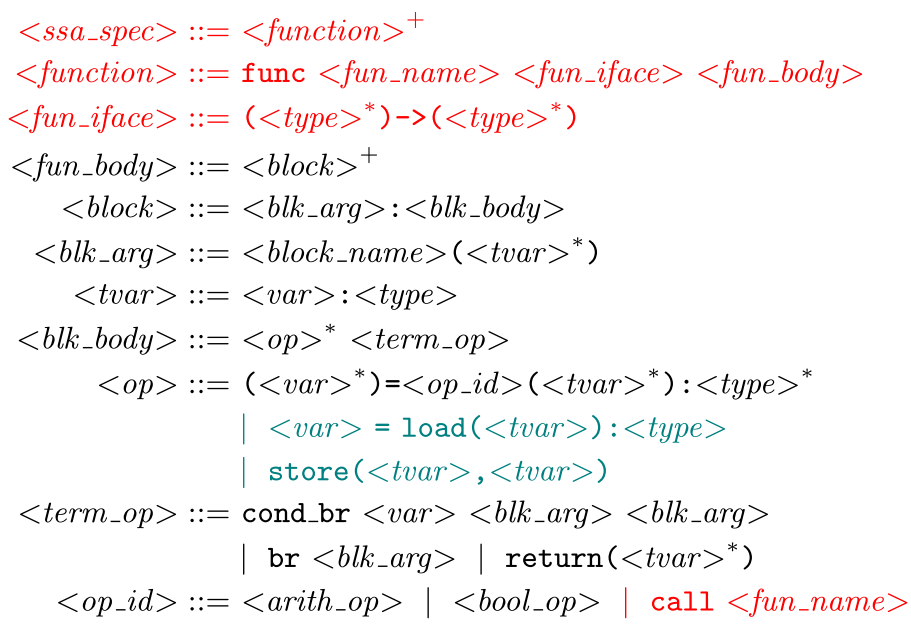

Fig. 1. SSA syntax.

\section{SSA SYNTAX AND SEMANTICS}

The syntax and semantics of SSA form have been presented multiple times before, but we need to settle on one as a basis for extension. The SSA variant we selected is based on MLIR [17], which uses continuation-passing style (CPS) branch arguments rather than $\phi$ operators.

Figure 1 presents a minimal SSA syntax (in black) extended with the constructs for functional composition (in red), and with the load and store operations to represent memory side effects (in green). ${ }^{2}$

Additional structural properties must be met. No two blocks of a function may have the same name, and branching operations (br and cond_br) may only reference existing blocks with the correct number of arguments. The blocks and the branching operations of an SSA function determine a sequential control flow graph (SCFG) structure, with the first block serving as control entry point. Execution proceeds sequentially inside each block. Each variable is either defined from one operation, or the argument of a single block header. ${ }^{3}$ No two functions may have the same name, and calling a function assumes that it exists and has the correct interface. The interface of a function consists in the arguments of its first block, which are also the function arguments, and the arguments of its return operation, which are the function return values. If multiple return operations exist in a function, then they must have the same number of arguments. All SSA blocks are terminated by a return or a branch operation. These operations are called terminators.

The small example of Figure 2 illustrates the specific properties of reactive systems and the differences between Lustre and SSA. The Lustre node (left) and MLIR SSA function (right) implement the same functionality, but we focus on the SSA function in this section. As we will see later, the latter could be the result of the compilation of the former. From now on, and to simplify the exposition, we use the input/output terminology of Lustre nodes for MLIR block and function arguments/return values. We will see that this terminology is well supported by semantics properties. Note that the MLIR SSA syntax requires that block identifiers start with "^" and that all

\footnotetext{
${ }^{2}$ The syntax of types is left implicit. The following semantics is largely independent on the type system for SSA expressions. ${ }^{3}$ Under classical SSA notation, this amounts to the variable being defined from exactly one $\phi$ operator. In classical SSA notation, $\phi$ operators are used in blocks that are destination of multiple branching operations. Each one allows the construction of a unique value based on values coming from the different source blocks.
} 


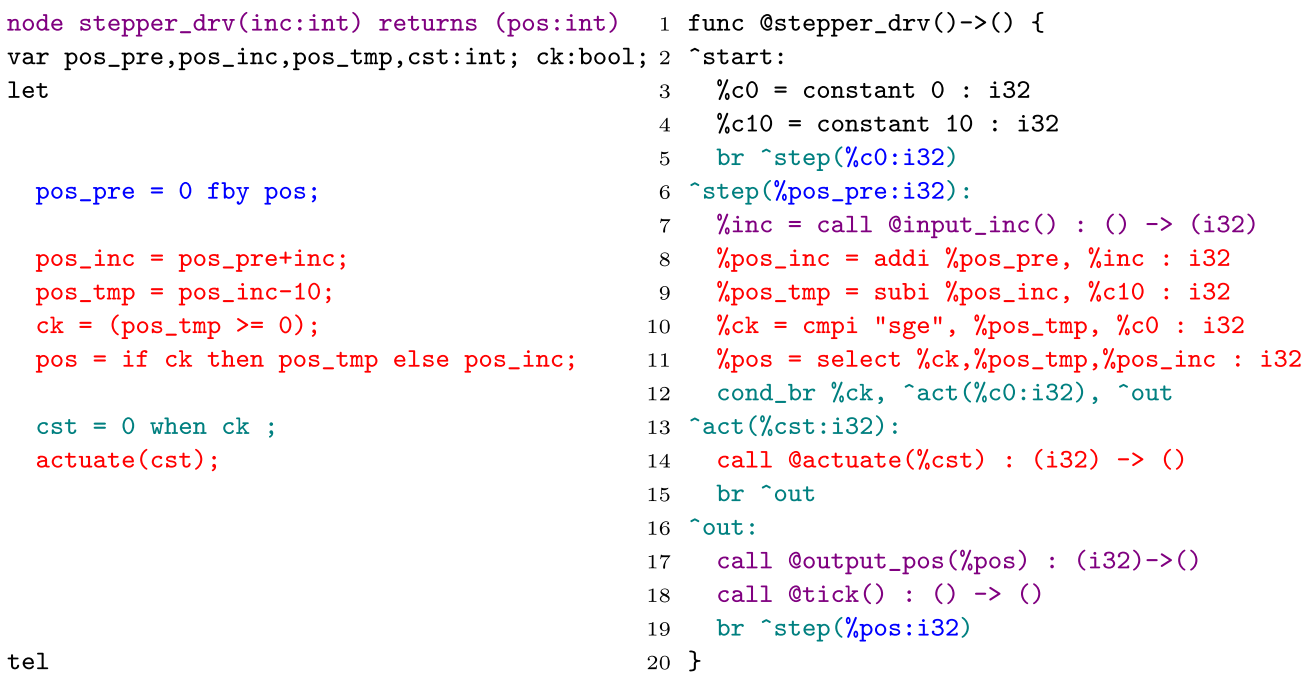

Fig. 2. Stepper motor driver in Lustre (left) and MLIR SSA (right). Control statements in green, data processing operations in red, cyclic $\mathrm{l} / \mathrm{O}$ and time synchronization in violet, state manipulation in blue.

variable names start with "\%"” MLIR also provides more intuitive forms for specific operations: For example, function calls specify the full function signature at their end; the comparison taking two input variables as arguments and producing one variable (the test result) has the syntax of line 10, which specifies the kind of test (sge for >=), the type of input data (i32), but not the type of the Boolean output, which is implicit (i1); operation select in line 11 outputs one of its data inputs based on the value of its Boolean test variable \%ck (of type i1).

Our example is a driver for a stepper motor, which receives rotation commands as increments of $0.18^{\circ}$, but can only actuate-issue physical commands to the motor-for $1.8^{\circ}$ at a time. For this reason, commands must be buffered and only actuated when their number exceeds 10 . This behavior, typical of an embedded control system, involves a continuous interaction with the environment. In our example, this interaction is driven by the infinite loop formed by the SSA branching operations. Each iteration of the loop is an execution cycle during which the program reads its input \%inc from its environment, performs computations, potentially actuates the motor and finally outputs \%pos. Timely interaction with the environment is achieved through the function calls in violet, which are discussed in Section 3.

\subsection{SSA Operational Semantics}

For conciseness, we shall assume all variables (including Booleans) are semantically represented as integers. We also assume each operation of the program has a unique label, such as a program line number if we assume that no two operations share the same line.

Notations. The cardinal of a set $\mathcal{S}$ is denoted $|\mathcal{S}|$. We use the OCaml notation for lists: [] is the empty list, $h:: t$ the list of first element $h$ and tail $t$.

Int is the domain of the integer type. To represent the status of a memory location or variable that has not been initialized, we use the special undefined value denoted $\perp$. Therefore, the status of any variable or memory location, at any execution point, is an element of $\overline{\operatorname{Int}}=\operatorname{Int} \cup\{\perp\}$. We also denote $\perp$ any function that always returns $\perp$, regardless of its domain. Given a (mathematical) 
function $f$ and a value $x$ of its domain, $f[x \leftarrow y]$ is the function that is identical with $f$ everywhere except on $x$, where it has value $y$.

$\mathcal{L}^{\mathrm{f}}$ and $\mathcal{V}^{f}$ are, respectively, the sets of labels and of variables of an SSA function $\mathrm{f}$, and $b_{0}^{f}$ is its first block. The function containing block $b$ is denoted $f u n(b)$. The ordered set of inputs of block $b$ is $\operatorname{in}(b) \subseteq \mathcal{V}^{f u n(b)}$, and $\operatorname{in}(b)_{i}$ is the $i$ th input of $b$. The ordered set of variables assigned by operations of a block $b$ is denoted $\operatorname{loc}(b)$. The label of the first operation in block $b$ is $f s t(b)$. The operation associated with a label $l \in \mathcal{L}^{f}$ is denoted $o p(l)$. If $o p(l)$ is not a terminator, then $n x t(l)$ is the label of the next operation in its block. The number of arguments of a return operation of function $\mathrm{f}$ is denoted $\mathrm{O}^{f}$.

Execution state. The execution state of an SSA function $f$ is one of:

- An initial state $\operatorname{Start}^{f}\left(v_{1}, \ldots, v_{\left|i n\left(b_{0}^{f}\right)\right|}\right)$, where $v_{i} \in \overline{\operatorname{Int}}$ are the actual parameters of the function.

- A final state $E n d^{f}\left(w_{1}, \ldots, w_{O}\right)$, where $w_{i} \in \overline{I n t}$ are the outputs of $f$ (received as input by return).

- A triple $\operatorname{Run}^{f}(p c, v a l)$ formed of the label $p c$ of the operation to execute next (the program counter) and a partial valuation val $: \mathcal{V}^{f} \rightarrow \overline{I n t}$ of all the variables.

The execution state of an SSA specification is a triple $(s, c s, m)$ formed of the state $s$ of the function that is currently executing, a list $c s$ of running states representing the call stack, and the current state $m:$ Int $\rightarrow \overline{\text { Int }}$ of the memory. An initial state of the specification has the form $\left(\operatorname{Start}^{f}(\ldots),[], m\right)$, where $m$ is the initial memory state and $f$ the function that serves as execution entry point. A final state of the specification has the form $\left(\operatorname{End}^{f}(\ldots),[], m\right)$.

Program execution. Transition rules are provided in Figure 3. An execution trace of an SSA specification is any sequence of transitions starting in an initial state. Note that if $t=s_{0} \rightarrow s_{1} \rightarrow \cdots \rightarrow$ $s_{n} \rightarrow \cdots$ is a trace, then any prefix $t_{n}=s_{0} \rightarrow s_{1} \rightarrow \cdots \rightarrow s_{n}$ is also a trace. We denote with $\leq$ the prefix order on traces, meaning we can write $t_{n} \leq t$.

Note that rules (ifthen) and (ifelse) define the behavior of a conditional branch only when the test variable is defined. When it is $\perp$, execution cannot advance-it blocks. Execution also blocks when a load or store operation accesses an address that is either $\perp$ or invalid. Under the LLVM interpretation of undefinedness [15], this amounts to assuming that the use of $\perp$ in decisions and computations is an immediate undefined behavior. To perfectly match this interpretation, we also

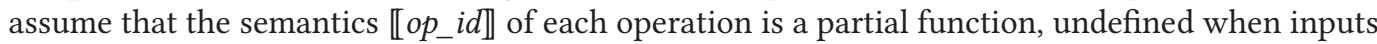
have value $\perp$.

Note that maximal traces (in the sense of $\leq$ ) will either be infinite (when execution never ends) or end in a final state, or end when execution blocks.

Separation assumptions. Our semantics separates, in both state expression and transition rules, the part corresponding to the SSA core from the extensions needed to represent function calls and memory. For instance, to consider only the core SSA semantics one simply has to remove call stack and memory terms from the specification state, and only consider the black rules of Figure 3 (which do not access memory or call stack). Similarly, function call rules do not access the memory, and memory rules do not access the call stack.

\subsection{Determinism and Correctness}

If we assume that all operations are deterministic (which in our formal framework amounts to

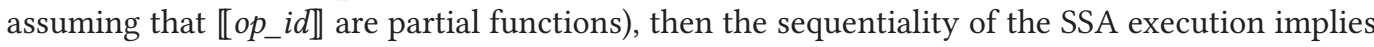




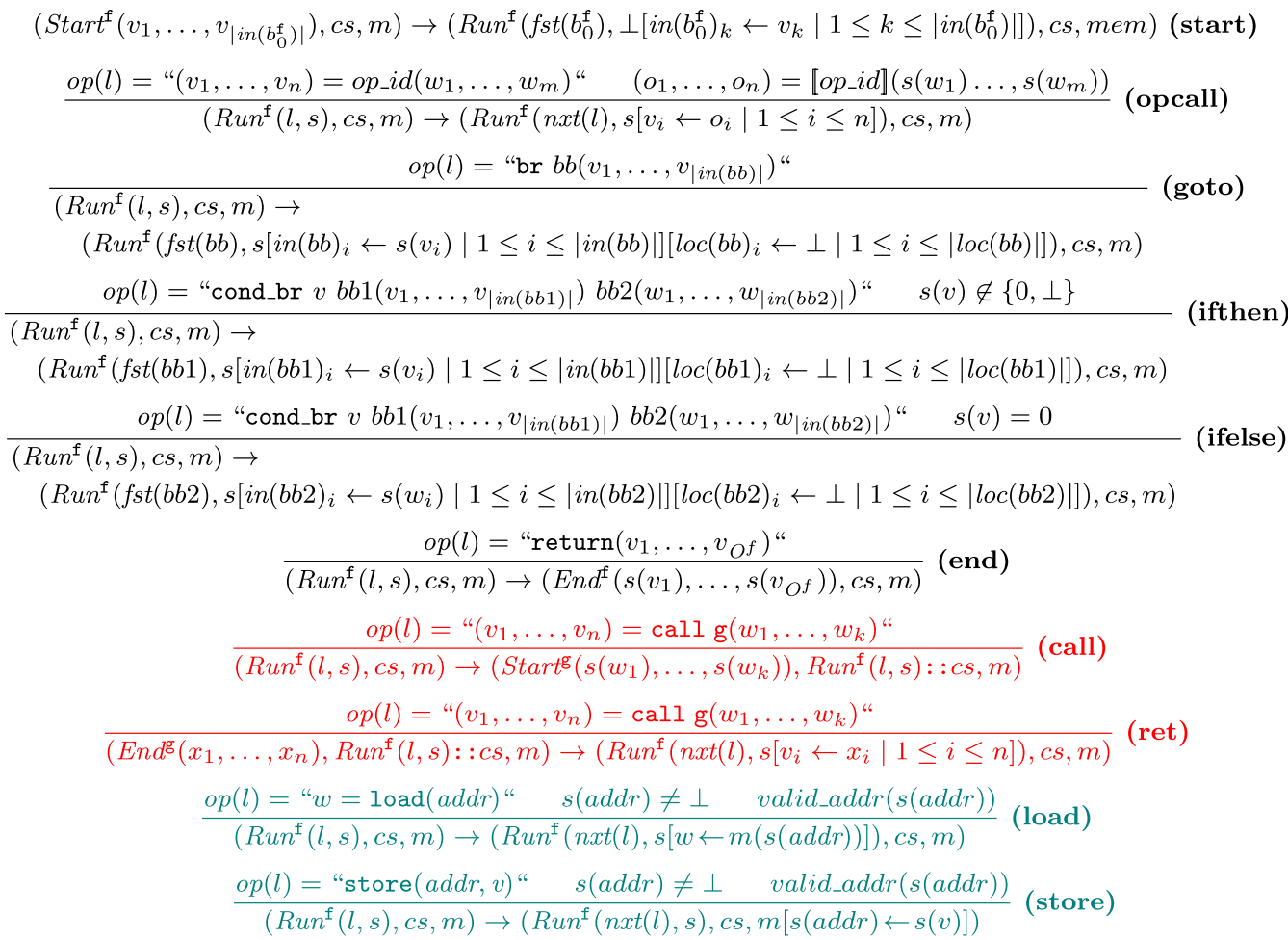

Fig. 3. SSA semantics. Memory access rules in green. Function call rules in red.

its determinism: For each initial state $s$ there exists a unique trace starting in $s$ that is maximal in the sense of $\leq$.

But determinism is not sufficient. Correctness also requires that execution never blocks. To ensure this, SSA enforces a stronger property: that all variables are different from $\perp$ whenever used in decisions, memory access adresses, and computations. Assume that variable $v$ is defined by block $b$, either by an operation of $b$ or by the block header itself. Also assume that $v$ is an input to operation $o^{\prime}$ of block $b^{\prime}$. To ensure that $v$ does not have value $\perp$ when $o^{\prime}$ is executed, one must ensure (as a necessary property) that any execution path reaching $o^{\prime}$ passes through the definition of $v$. This happens if and only if one of the following conditions is true:

D1 $b=b^{\prime}$, and the definition of $v$ precedes $o^{\prime}$ in $b$, either as block argument, or as output of an operation.

D2 $b \neq b^{\prime}$, and any possible execution reaching $b^{\prime}$ necessarily passes through $b$.

While checking property $\mathbf{D} 1$ is straightforward, determining that D2 holds for any variable $v$ and operation $o$ using $v$ is not tractable in the general case (Boolean satisfiability can be reduced to the decision of D2).

For this reason, SSA-based compilation always ensures D2 by enforcing a sufficient property, named dominance, which can be checked using a low-complexity structural analysis of the SSA SCFG [8]. Dominance is considered part of SSA-form correctness, along with the syntactic correctness and the structural properties of Section 2. 
Together, these structural properties ensure the correctness of SSA specifications that do not access memory. When memory is used, these properties must be complemented by a proof of the fact that each memory location is initialized before it is read.

\section{FROM SSA TO SYNCHRONOUS CONCURRENCY}

In this section, we extend the syntax and semantics of SSA with the operational mechanisms needed to represent synchronous concurrency. The extension leaves the (non-reactive) SSA semantics of Figure 3 unchanged, and allows the application of all SSA code transformations. Thus, building on MLIR's extensible syntax and semantics, it is possible to model and generate code for reactive real-time applications without changing the existing code base (only introducing additional behaviors).

\subsection{Cyclic Execution}

To represent the behavior of embedded systems, which interact with their environment in a continual and timely fashion, all synchronous languages have cyclic execution models. The execution of a synchronous program is an a priori infinite sequence of execution cycles. Execution cycles being non-overlapping, they form a logical time base: each operation happens in exactly one logical cycle, which can be identified by its index. But synchronous logical time is not only a descriptive notion used during analysis. It is meant to allow the synchronization of cycle execution onto external time bases. For instance, periodic real-time execution is typically enforced by synchronizing cycle triggering (the logical time base) with a periodic HW timer.

The SSA form also allows the representation of cyclic behaviors under the form of cyclic CFGs, as exemplified in Figure 2. However, what constitutes an execution cycle is not clearly identified, nor the mechanisms for synchronizing execution cycles with an external time base, nor how to constrain an operation to happen in a specific cycle. To allow SSA-based embedded real-time programming, we must allow the specification of all these aspects in a way that will be preserved by SSA-based code transformations and optimizations.

Tying the definition of execution cycles to the structural elements of SSA ${ }^{4}$ is tempting, but unfeasible, as basic blocks are not preserved by common optimizations. Another tempting approach is to extend the syntax and semantics of basic blocks and/or branching statements to explicitly identify some of them as execution cycle barriers. However, such an approach would require changes to much of existing SSA-related compiler code (such as dominance analysis or optimization algorithms), which we want to avoid.

3.1.1 The tick Operation. The solution we chose is the introduction of a new operation tick to identify execution cycle barriers. To order operations with respect to these barriers, tick relies on two SSA mechanisms: dominance and memory access ordering.

Dominance-based ordering. The syntax of tick, provided in Figure 4, shows that it can take as arguments any number of variables, which allows specifying that operations producing these variables are executed before the cycle barrier. It produces an optional variable \%s of type none, which is the unit type of MLIR SSA.

Like for the unit type of functional programming or the pure signals of synchronous programming [19], a variable of type none represents pure synchronization. It carries no information but requires operations reading it to happen after the operation producing it. To facilitate the specification of such ordering constraints, we also introduce the sync operation, which allows transferring

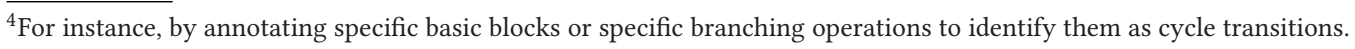



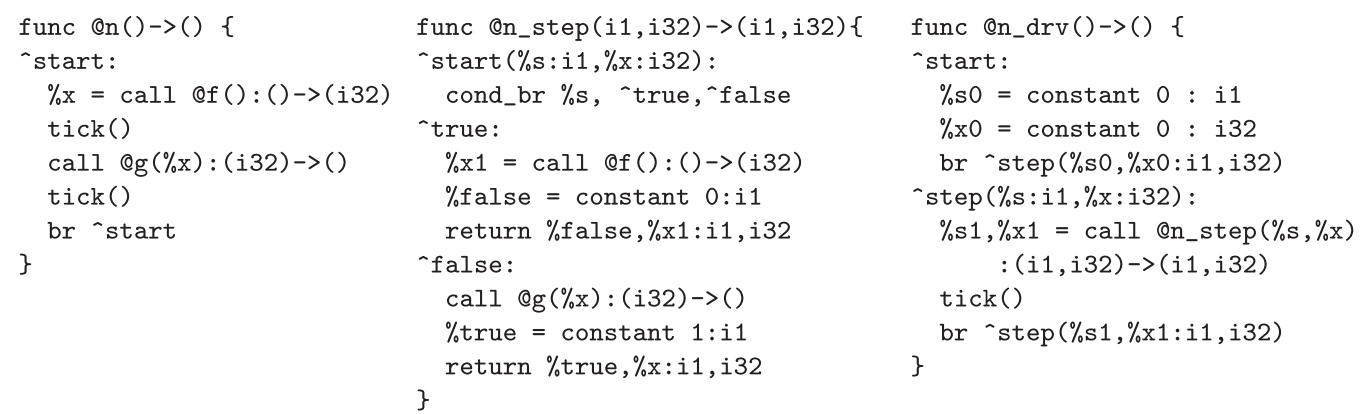

Fig. 6. Synchronous languages approach to code generation. Source code left, output middle and right.

function between execution cycles must be explicitly represented. In our example, this state is transmitted by the arguments of ^step and consists of:

- A Boolean value (of type i1) to determine whether @f or @g should execute in the next cycle.

- A value of type i32 allowing the transmission of the output of af to @g.

At the beginning of each cycle the state is decoded, the needed computations are triggered, then a new state is encoded and transmitted to the next cycle. This process is usually represented with a separate step function, in our case @n_step.

Classical synchronous language compilers will usually produce just this step function and the data structure describing the application state. When used in conjunction with the dataflow modularity described in Section 3.3, this approach allows modular code generation ${ }^{5}$ [4]. However, the driver function (in our case en_drv), and in particular the implementation of tick, are usually not generated, being considered too implementation-dependent.

This compilation approach has been long tested in practice [2, 12], where it has shown its strengths (most notably modularity) but also its limits. The limits are mainly related to the onesize-fits-all generated code with a single step function and a state representation that must cover the needs of all execution cycle transitions. In our example, in the compilation output, the state variable of type $i 32$ is assigned a value and then passed on to the next cycle in every cycle, even though it is semantically produced (output of @f) only in odd cycles. A first evaluation of the inefficiency of the inverted state representation is provided in previous work on state representation optimization for the Esterel language [19]. ${ }^{6}$ It is important to note that, once the generation of the step function performed, classical compiler optimizations are confined to the scope of the step function, and optimizations involving multiple execution cycles must be specifically designed for each particular language and state encoding.

Our compilation method cannot follow this example and systematically restructure control to obtain a loop with a single tick. Not only because of the potential efficiency loss, but because in many cases the implementation must have a different structure. For instance, in avionics MIF/MAF applications [10] the implementation must have a structure similar to that of Figure 6 (left), where a global periodic pattern (the global loop, named major frame, or MAF) is split by the tick operations into time intervals of equal length (the minor frames, or MIFs), each containing a different code.

\footnotetext{
${ }^{5}$ One step function per hierarchic synchronous module, the state representation of a module including that of sub-modules it hierarchically includes, and its step function calling the step functions of sub-modules.

${ }^{6}$ Memory allocation for synchronous languages also poses other challenges, such as the fact that modular code generation requires in the general case an inefficient encoding for memory constructs, which uses not one, but two variables for each state element. Several papers address this issue in previous work [10, 11].
} 
To allow the implementation, without restructuring, of any reactive SSA graph satisfying the structural properties defined above, we propose a return to the fundamentals of reactive systems design, by making explicit the interaction with the system scheduler. In our compilation approach, a reactive SSA specification such as function @n of Figure 6 (left) is seen as a sequential process running under a cooperative multi-tasking scheduler. Each time the execution of @n reaches a tick operation, the execution context (state) of an is saved and control is given back to the scheduler When the scheduler determines that a new execution cycle must be triggered, it restores the state of the process, execution of the tick operation terminates, and the scheduler hands returns control to the process.

This operational mechanism, whose formal semantics is defined in Section 3.5, can be easily implemented on various execution platforms ranging from low-level timers on bare metal platforms [10] to POSIX system services such as longjmp (as we do in Section 5) to coroutine mechanisms of various languages and to RTOS services such as PERIODIC_WAIT in the avionics-oriented IMA/ARINC 653 standard [1].

Note that our proposal does not exclude the classical approach of compiling synchronous languages. Instead, it is complementary, allowing the modeling of implementation aspects that were previously not covered by code generation, and by allowing more expressiveness in the implementation.

\subsection{Cyclic I/O}

An embedded system will continually interact with its environment, cyclically reading inputs and writing outputs. In practical implementations, this is typically done by reading and writing memory-mapped registers that can be represented with volatile $\mathrm{C}$ variables, or by calling dedicated I/O functions. Synchronous languages abstract away such implementation-dependent mechanisms under the form of input and output variables that can be read or written at each cycle, with two constraints related to the synchronous model:

- An output variable can be written at most once per execution cycle.

- All reads of an input variable during an execution cycle must return the same result.

For instance, in Figure 2, the input inc of the Lustre program is read at each execution cycle.

By comparison, when not considering memory side effects, MLIR functions interact with their environment only twice:

- At the beginning of their execution, to read the value of their input arguments, which then remains constant during the execution of the function.

- When reaching a return operation, when the function completes.

These assumptions enable common SSA optimizations such as loop-invariant code motion. Volatile accesses to memory locations can sometimes be represented, like in the low level dialects of MLIR (LLVM, SPIRV), but they represent a particular low-level implementation, excluding others.

The solution we chose to represent cyclic I/O is based on the representation of input and output channels with function arguments of the special types in $(t)$ and out $(t)$, where $t$ is the type of data transmitted by the channel. Access to channel variables is done exclusively using the input and output operations, whose syntax is provided in Figure 4. Operation input has a single argument of input channel type. Each time it is executed, input samples the channel for a value of the correct type, which is placed in the output variable. An output operation has two inputs: one output channel and a second variable (of the corresponding non-channel type) whose value will be written to the output. 

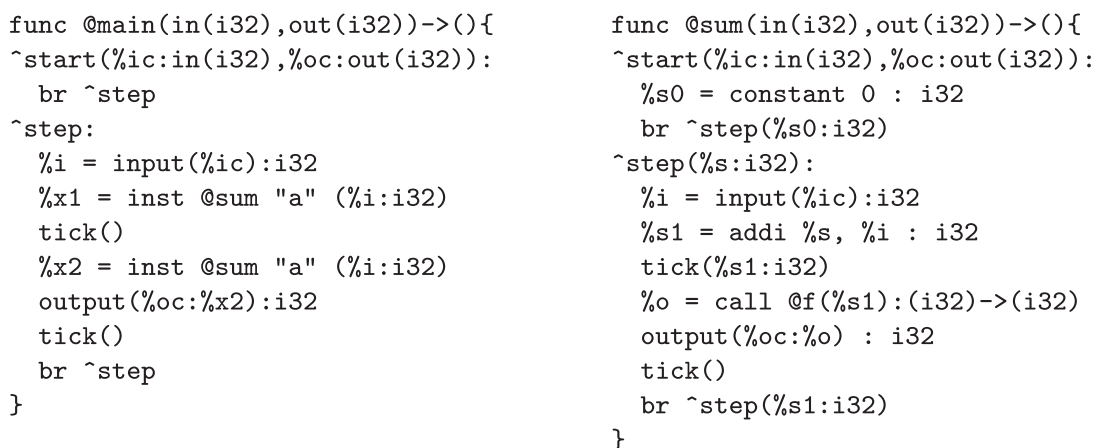

Fig. 8. Submodule instantiation example.

to an output operation are allocated and deallocated by the function, and that the environment no longer uses them when the next execution cycle begins.

\subsection{Modularity}

The modularity of SSA is that of sequential procedural programming. The modules of an SSA specification are the functions, which interact through function calls.

By comparison, the formal models underpinning synchronous languages are concurrent. In the most general settings, such as Esterel's constructive semantics [19], the execution of two submodules of a specification can advance concurrently, synchronizing and communicating with each other in both ways. Determining that the execution of such a specification does not block (a process known as causality analysis) is in general undecidable, if integer data are allowed, and NP-hard (untractable in practice) if the input language uses only Boolean variables. Furthermore, the implementation of such general specifications can be very inefficient due to intricate semantic rules.

For this reason, synchronous language compilers have early on imposed simple structural constraints allowing fast and modular compilation: in each module, the computations of an execution cycle must form an acyclic dependency graph, allowing fast scheduling and code generation. In this acyclic graph, to allow the separate compilation of a sub-module, its computations must be grouped together as a single graph node, meaning that they can be performed atomically.

To allow the representation of this mechanism in our SSA extension, we introduce the notion of instance of a reactive function @f, which is a process (with separate state) executing function $\% f$ under the system scheduler. Instances are uniquely identified (with lists of strings, in our implementation). We assume that the first reactive function of a specification has an instance identified with the empty list of strings [] that receives control when the system starts. All other instances are inductively defined and possibly given control during execution using the operation inst (syntax in Figure 4): if $i$ is an instance of function @f, which contains operation "inst @g str," then instance str: : $i$ of function $Q g$ is automatically defined.

We provide in Figure 8 an example of submodule instantiation. The system has two reactive functions and two instances: the implicit [] instance of function @main and one instance ["a"] of function @sum. Notice that the two inst operations of @sum trigger execution cycles of the same instance $([" \mathrm{a} "]) .^{8}$ Instance [] reads its unique input from the environment on odd cycles, triggers one tick of instance ["a"] in every cycle (giving it as input the value of \%i) and outputs the last

\footnotetext{
${ }^{8}$ In the presence of periodic activation patterns, this enables the unrolling of the infinite loop over the period, followed by constant propagation, which eliminates activation tests over the iteration counter.
} 
output of ["a"] in even cycles. Instance ["a"] of @sum has a state in which it accumulates the sum of inputs it receives on odd cycles. On even cycles, it computes and outputs variable \%o.

Structural constraints. The number and types of input and output variables of an inst operation must match the signature of the reactive function it instantiates.

Compilation. The compilation method based on conversion to step functions will implement submodule instantiation using function calls [4]. When conversion to a step function is not desired, we transform each instance into a process running under a cooperative scheduler. This mechanism extends that of Section 3.1.2 by clarifying how the scheduler passes the control between instances. When reaching an inst operation, the inputs and the control are transmitted to the instance, which then executes until reaching a tick operation, at which point it saves its state and returns the outputs to the caller, according to the rules of Section 3.5.

\subsection{Signal Absence}

Consider the simple example in Figure 6 (left) and its implementation on the right. Note that the translation slightly changes the internal behavior of the program: on the left, the variable $\% \mathrm{x}$ is transmitted only from odd cycles to even cycles. But in the translation result \%x has been added to the program state, which is computed and transmitted at every cycle. Thus, in the ${ }^{\wedge} \mathrm{false}$ : basic block of @n_step, under SSA semantics, we need to return a value for \%x at each cycle, even if the source program does not require it.

In this case, we made the natural choice of maintaining the previous value, which later allows the encoding of the state in (persistent) memory. But using instead a constant of type i32 or a dynamically computed value would also have been correct, as this value is never used in computations. The situation is similar in Figure 8: function @sum outputs values only on even cycles, meaning that the value of \%x1 in function @main is never correctly initialized, yet the specification is overall deterministic, because this absent/undefined value is never used in computations.

Such situations are common in the synchronous modeling of multi-rate and multi-periodic systems, which explains why absence prominently figures in the semantics of all synchronous languages [2]. As we shall see in Section 4, in the Lustre language a variable that is absent in a synchronous execution cycle has an undefined value and cannot be used in decisions or computations. This definition fully matches that of $\perp$ in Section 2.1.

However, synchronous languages such as Lustre are designed to support low-complexity analyses (known as clock calculi) ensuring that no uninitialized data is ever used in computations (much like the SSA form is designed to support dominance analysis). These analyses exploit the tight coupling between (dataflow) control propagation and initialization, as well as stringent synchronization requirements (known as clock constraints) enforced by each language primitive.

But our reactive extension of SSA is meant to be an intermediate compiler representation, where efficient encodings may require the explicit representation and manipulation of absence/ undefinedness, which breaks the coupling between SSA control propagation and initialization. This situation is similar to that of LLVM IR, which extends the basic SSA syntax and semantics with not one, but two types of undefinedness to support different analysis and optimization approaches$1 \mathrm{lvm}$. undef and llvm. poison [15].

Unlike LLVM IR, we do not need to add a new semantic value. Instead, we simply allow the explicit manipulation of $\perp$ without breaking the structural correctness rules of SSA. To do this, we introduce operation undef (syntax in Figure 4, semantics in Figure 9). This operation sets its output variable to $\perp$. To understand how this value is propagated, consider function @n_step of Figure 6, and recall that in this function, in the ${ }^{\wedge}$ false basic block, the second argument of return could take any value instead of \%x, because it is never used in computations. Using operation undef, we 


$$
\begin{aligned}
& \frac{\mathcal{I}(i)=(s, c s, s i, s o) \quad(s, c s, m) \rightarrow\left(s^{\prime}, c s^{\prime}, m^{\prime}\right)}{<i, \mathcal{I}, m>\Rightarrow<i, \mathcal{I}\left[i \leftarrow\left(s^{\prime}, c s^{\prime}, s i, s o\right)\right], m^{\prime}>} \text { (local) } \\
& \frac{\mathcal{I}(i)=\left(\operatorname{Run}^{\mathrm{f}}(l, s), c s, s i, s o\right) \quad o p(l)=\text { " } v=\operatorname{input}(i c) \text { " }}{<i, \mathcal{I}, m>\Rightarrow<i, \mathcal{I}\left[i \leftarrow\left(\operatorname{Run}^{\mathrm{f}}(n x t(l), s[v \leftarrow s i(i c)]), c s, s i, s o\right)\right], m>} \text { (in) } \\
& \frac{i=[] \quad \mathcal{I}(i)=\left(\operatorname{Run}^{\mathrm{f}}(l, s), c s, s i, s o\right) \quad o p(l)=\text { "tick" }}{<i, \mathcal{I}, m>\stackrel{s o}{\underset{s i^{\prime}}{\longrightarrow}}<i, \mathcal{I}\left[i \leftarrow\left(\operatorname{Run}^{\mathrm{f}}(\operatorname{nxt}(l), s), c s, s i^{\prime}, \perp\right)\right], m>} \text { (system-tick) }
\end{aligned}
$$

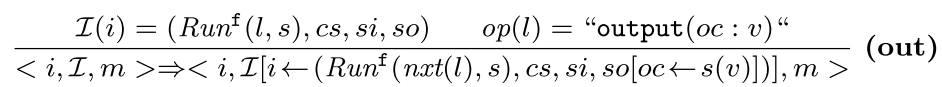

$$
\begin{aligned}
& \mathcal{I}(i)=\left(\operatorname{Run}^{\mathrm{f}}(l, s), c s, s i, s o\right) \quad o p(l)="\left(v_{1}, \ldots, v_{n}\right)=\text { inst nd } i^{\prime}\left(w_{1}, \ldots, w_{k}\right) " \\
& \mathcal{I}\left(i^{\prime}:: i\right)=\left(\operatorname{Run} \mathrm{g}\left(l^{\prime}, s^{\prime}\right), c s^{\prime}, s i^{\prime}, s o^{\prime}\right) \\
& <i, \mathcal{I}, m>\Rightarrow<i^{\prime}:: i, \mathcal{I}\left[\left(i^{\prime}:: i\right) \leftarrow\left(R u n^{\mathrm{g}}\left(l^{\prime}, s^{\prime}\right), c s^{\prime}, \perp\left[i n_{l}^{\text {nd }} \leftarrow s\left(w_{l}\right) \mid 1 \leq l \leq k\right], \perp\right)\right], m> \\
& \mathcal{I}\left(i^{\prime}:: i\right)=\left(R u n^{\mathrm{g}}\left(l^{\prime}, s^{\prime}\right), c s^{\prime}, s i^{\prime}, s o^{\prime}\right) \quad o p\left(l^{\prime}\right)=\text { "tick" } \\
& \frac{\mathcal{I}(i)=\left(\operatorname{Run}^{\mathrm{f}}(l, s), c s, \text { si, so }\right) \quad o p(l)="\left(v_{1}, \ldots, v_{n}\right)=\text { inst nd } i^{\prime}\left(w_{1}, \ldots, w_{m}\right) "}{<i^{\prime}:: i, \mathcal{I}, m>\Rightarrow<i, \mathcal{I}\left[i \leftarrow\left(\operatorname{Run}^{f}\left(l, s\left[v_{i} \leftarrow s o_{i}^{\prime} \mid 1 \leq i \leq n\right]\right), c s, \text { si, so }\right)\right], m>} \text { (inst-tick) }
\end{aligned}
$$

Fig. 11. SSA semantics extension for reactive synchronous systems. In red, modularity rules.

values or with $1 \mathrm{lvm}$. undef or $1 \mathrm{lvm}$. poison. These differences can only be propagated through assignment, as they do not reach decisions, memory access addresses, or computations.

\subsection{Formal Semantics of Reactive Extensions}

The final step of our SSA extension is the definition of the formal semantics of reactive SSA specifications formed of one or more instances of reactive SSA functions.

Given a reactive function $f$, we denote with $i n^{f}$, respectively, out ${ }^{f}$ the ordered set of input channel variables of $\mathrm{f}$. Given an instance $i$, we denote with $r(i)$ its reactive function.

The execution state of a reactive SSA specification is represented with triples $\langle i, \mathcal{I}, m\rangle$, where $m$ is the shared memory state, $i$ is the currently active instance identifier, and $\mathcal{I}$ is a map associating to each instance identifier the instance state. The state of instance $i$ is $\mathcal{I}(i)=(s, c s, s i, s o)$, where $s$ is the state of the function $\mathrm{f}$ that is currently executing, $c s$ is the call context, $s i: i^{r(i)} \rightarrow \overline{\operatorname{Int}}$ is the state of the input channels of $i$, and so $:$ out $^{r(i)} \rightarrow \overline{\operatorname{Int}}$ is the state of the output channels.

Under these notations, the operational semantic rules are provided in Figure 11. The (local) rule transforms non-reactive SSA transitions of the instances (denoted with $\rightarrow$ and defined in Figures 3 and 9) into transitions (denoted with $\Rightarrow$ ) of the reactive system. All other rules involve reactive operations (interaction with the scheduler). Rules (in) and (out) deal with instance I/O. Rule (system-tick) is the only one that interacts with the environment by performing I/O and possibly time synchronization. The rules (inst) and (inst-tick) (in red) implement modularity. The first one gives control to an instance when reaching an inst operation, and the second one recovers control from it when the execution of the instance reaches a tick operation.

Compilation correctness issues. While defining a formal semantics for reactive SSA specifications, this article does not provide a formal proof of the fact that their compilation to executable code is correct. One issue here is proving that the primitives of the sync dialect work as intended. In turn, this requires modeling the target execution platform, which may range from bare hardware to real-time operating systems.

But even assuming that the primitives are correctly implemented, we still have to prove that correct SSA code transformations preserve the semantics of reactive specifications. This is a key issue, because the whole point of our work is to allow existing SSA code transformations and optimizations to be applied at full strength in the compilation of reactive programs. 
This problem is difficult and involves multiple difficult sub-problems. For instance, while we provide a formal semantics for reactive SSA specifications, this semantics does not cover MLIRspecific extensions to SSA. To understand the importance of these extensions, consider the tick() operation. As explained in Section 3.1.1, this operation has unspecified side effects. This is why correct SSA code transformations cannot make function calls ${ }^{11}$ commute with tick() operations, and thus cannot change the allocation of function calls into execution cycles.

Side effects are specified in MLIR using operation properties called traits. These MLIR-specific constructs must be taken into account by any formalization of the correctness of the SSA code transformations. In other terms, our formalization of the SSA semantics must be extended, if the objective is to formally state or prove the correctness of any MLIR-based compilation process (including ours).

However, even though formal arguments are missing, we consider that we have provided initial evidence that existing SSA code transformations and optimizations can be applied at their full strength in the compilation of reactive specifications.

The first argument supporting this statement is that the (complex) use cases of Section 5 run as expected after multiple, complex MLIR code transformations and optimizations. This form of validation is akin to the traditional regression testing used to validate most compilers (MLIR included), and we must significantly extend it.

The second type of evidence consists in the careful choices we explicitly made in Section 3 in the definition of the sync dialect operations (e.g., the fact that tick() has unspecified side effects). Along with the existing SSA semantics, these choices provide support for both semi-formal reasoning, and for the future formalization of the MLIR SSA extensions.

\section{EMBEDDING LUSTRE IN MLIR}

In the previous section, we have extended the SSA form with the features allowing the representation of synchronous reactive behaviors. Our objective was to do this in the most general way that remains fully compatible with traditional SSA semantics and algorithmics (thus allowing implementation without changes to the existing codebase).

In this section, we evaluate the ability of this extension to support the specification and compilation of realistic applications with both reactive and HPC aspects. The reactive aspects of such applications must be specified in a high-level synchronous dialect, not directly in our low-level SSA extension. We show that the dataflow core of the Lustre synchronous language ${ }^{12}$ can be embedded as a new dialect, named lus, into the SSA-based MLIR compilation framework [17]. This allows the specification (in MLIR) of applications where the reactive aspects are modeled at the Lustre abstraction level, while data processing is modeled at the abstraction level of other dialects such as affine (affine loops), linalg (linear algebra), or tf (TensorFlow graphs).

During compilation, reactive statements of the lus dialect are lowere $d^{13}$ into a mix of structured control flow (MLIR dialect SCf) and the reactive SSA constructs introduced in Section 3 (Figure 4) and grouped in a new dialect named sync. Operations of the sync dialect are later converted into low-level SSA (dialects std and $1 \mathrm{lvm}$ ) and calls to external OS primitives following the compilation rules defined in Section 3.

The structured control flow, along with the data types and data processing code, are progressively lowered using the existing transformations of MLIR, which do not affect reactive semantics. Among others, these transformations allow buffer synthesis, i.e., the transformation of the abstract

\footnotetext{
${ }^{11}$ Which implicitly have side effects in MLIR.

${ }^{12}$ Chosen for its practical importance, as well as for its simplicity.

${ }^{13}$ That is, transformed into code at a lower abstraction level.
} 
aggregate data used with lus-level synchronous specification (e.g., tensors) into memory objects along with allocation and deallocation operations, in a way that ensures the absence of both memory leaks and accesses to unallocated memory.

The result is a fully functional specification and compilation framework for reactive high-performance applications, which we evaluate in the next section on three non-trivial applications (a signal processing vocoder and two machine learning applications).

\subsection{The Lustre Language}

The synchronous language that has reached the most widespread use is Lustre $[3,12]$. For space reasons, we only consider here its pure dataflow core-the SN-Lustre dialect of Reference [6]-into which full Lustre can be translated.

A Lustre program, like that of Figure 2 (left), is called a node. It specifies a dataflow graph of statements connected through dataflow variables. Each variable is either an input of the node, or it is output of exactly one statement (single assignment property). Lustre follows a pure dataflow paradigm, with no use of load/store memory (no side effects).

The semantics of Lustre is (intuitively) best described as a mix of dataflow operational and declarative aspects.

4.1.1 Operational Interpretation. As synchronous programs, Lustre nodes have a cyclic execution model. At each execution cycle, the list of statements of a node is traversed once, in an order compatible with the dependences determined by the variables. When traversed, a statement will read the value of its input variables, possibly perform some internal computations, and assign a value to its output variables. As the semantics of a node is not affected by the syntactic order of its statements, we can always assume (as a normal form assumption) that the statements already are in traversal order, as in Figure 2 (left).

Dependences are defined as follows: Variable y being produced by statement $p$ and used in statement $c$ determines a dependency $p \rightarrow c$ in all cases except one: when $c$ is a statement of the form " $\mathrm{x}=\mathrm{k}$ fby $\mathrm{y}$." In this case, $c \rightarrow p$, as a form of anti-dependency ensuring that the value is read before being overwritten. This special handling of fby allows it to read the value assigned to its input variable in the previous execution cycle. This value is then assigned to the output of fby, allowing its use in the current cycle. In Lustre, this is the only mechanism allowing the specification of a state passed between execution cycles.

The value assigned to a variable in a cycle can be the special value $\perp$ introduced in Section 2.1 to represent undefinedness/absence. Making a variable absent inside an execution cycle is done using the sub-sampling statement when. Traversing statement " $\mathrm{x}=\mathrm{y}$ when $\mathrm{c}$ " while the Boolean variable $\mathrm{c}$ has value true results in $\mathrm{x}$ being assigned the value of $\mathrm{y}$. If $\mathrm{c}$ is false or $\perp$, then $\mathrm{x}$ is assigned value $\perp$. Absence is the dataflow mechanism allowing the specification of conditional execution: A function call with $\perp$ input variables will not perform computations, but instead assign all outputs to $\perp$. This mechanism, specific to the dataflow programming paradigm, is used in lines 13 and 14 of Figure 2 (left) to specify that function actuate is executed only in cycles where variable ck is true. The counterpart of when is statement " $x=$ merge c y $z$ ". Upon traversal, if $c$ is true (respectively, false), $x$ takes the value of $y$ (respectively, $z$ ). Otherwise, $x$ takes the value $\perp$.

4.1.2 Declarative Aspects (Clock Constraints). The purely operational interpretation defined above matches the standard Lustre semantics $[3,4,6]$ on correct Lustre programs where all fby statements are executed at every cycle.

However, Lustre allows a form of logical time modularity during specification, by allowing subsets of statements (including fby statements) to be executed under specific Boolean conditions. This is the case in the node of Figure 12 (left), which incrementally computes the sum of its positive 
The MLIR definition of nodes is derived from that of SSA functions by replacing keyword func with node and requiring the compiler to check that a single basic block is present, terminated with operation lus.yield (which identifies the output variables).

While MLIR is SSA-based and its transformations mostly require the respect of dominance, it also allows specification under the weaker single assignment property, thus allowing the representation of cyclic dataflow graphs like the one in Figure 13 (left).

At the level of lus, we have implemented the Lustre clock inference algorithm. Along with data type correctness, single assignment, and causal correctness, the success of clock inference guarantees the correctness of a Lustre program. As data type correctness and single assignment are automatically checked by the existing MLIR infrastructure, only causal correctness remains to be checked.

4.2.1 Normalization. Once clock inference performed, we can transform the original program so that the operational interpretation correctly simulates it. This transformation, exemplified in Figure 12, consists in ensuring that all fby statements are executed at every cycle (through the introduction of when and merge statements, in red in Figure 12). We have implemented this normalization step in MLIR.

4.2.2 Lowering of lus to sync. After normalization, moving from the dataflow style of lus to the control-flow style of the sync dialect of Section 3 (Figure 4) is done in two steps, as exemplified in Figure 13:

- Moving from dataflow node-based modularity to control-flow function-based modularity.

- Encoding of conditional execution and absence.

The first step starts by collectively replacing all fby operations with the continuation passing encoding of state specific to MLIR SSA. In Figure 13 (middle) this is done using the basic branching mechanism of SSA, but the lowering phase we implemented in MLIR produces structured control flow operations (for, of dialect scf). Once the state reencoding performed, determining the causal correctness of the program amounts to an SSA dominance check, performed automatically by MLIR.

Then, the I/O variables of the lus node are transformed into I/O channels and explicit input and output operations, and the node interface is transformed into a function interface. This concludes the first lowering step.

Once the first step is done, the semantic rules of Figures 3, 9 and 11 can already be applied, and the need to re-encode signal absence becomes evident. Indeed, in the definition of the Lustre semantics, we have made the implicit assumption that operation call semantics is changed to produce $\perp$ output upon receiving $\perp$ input (and not block execution). This is acceptable from a semantic point of view (the Merge operation of TensorFlow already uses a similar mechanism [22]). However, from an implementation standpoint this would require wrapping every data type to account for the extra $\perp$ value, and wrapping function to ensure that undefined inputs result in absence of execution. To avoid this (for efficiency), the dataflow control transmitted by the $\perp$ values is materialized under the form of branching code that determines, based on the value of clock variables (e.g., \%ck in Figure 13) whether operations are executed or not. In Figure 13 (right) this imperative control is implemented using core SSA branching statements, but our MLIR implementation uses structured control flow operations.

The clock analysis of the Lustre program ensures that the resulting SSA specification cannot block under blocking semantics due to operations receiving undefined values ${ }^{15}$; proving this result

\footnotetext{
${ }^{15}$ They can still block due to an operation being undefined for the provided values, e.g., division by zero.
} 
goes beyond the scope of this article, but the reader may refer to References $[3,4]$ for formalization and proofs in the classical Lustre setting.

This completes the definition of our compilation method. Note that, in the implementation of our compiler, stock MLIR algorithms are used for all data type specification and implementation, causality analysis, and memory allocation. We have only had to implement analysis and lowering steps specific to the synchronous model of computation-clock analysis, normalization, and the lowering of normalized lus to sync.

\section{USE CASES}

In this section, we use three complex applications to illustrate the expressiveness of our MLIR embedding of Lustre, and to evaluate its effectiveness. The applications have been selected to cover various profiles involving both performance-intensive and reactive aspects:

Vocoder is a real-time sound processing application (a voice pitch tuning vocoder). This application, which relies on classical signal processing algorithms like the Fast Fourier Transform (FFT) is representative for traditional performance-intensive signal processing applications. It also features complex reactive control: a sliding window over the inputs, a keyboard-driven human-computer interface allowing muting the sound output and changing its volume, and a stateful signal processing algorithm.

TimeSeries is a recurrent neural network based on LSTM [14]. To faithfully implement the LSTM stateful behavior in a streaming application, this application features multi-rate execution. This application is representative for convolutional neural networks with complex control.

Resnet50 is an instance of the classical ResidualNet [13], representative for large feedforward deep neural networks.

All three applications have non-trivial data processing pipelines and are naturally expressed using the reactive primitives of the lus dialect and the data-processing features of existing MLIR dialects.

To evaluate the expressiveness of our MLIR embedding of Lustre we show that, for all three use cases, MLIR extended with the lus dialect allows the natural and concise expression of all application aspects (data processing and reactive ones). This means that the modeling approach we propose is more adapted to high-performance embedded applications than either MLIR alone (which lacks reactive specification constructs) and various dialects of Lustre, which lack advanced tensorial data specification and manipulation routines, as well as the efficient compilation apparatus.

Part of the effectiveness evaluation has been done in the previous section, which showed that the few Lustre-specific compilation algorithms (clock analysis, normalization, lowering of lus to sync) can be embedded into MLIR, and that stock MLIR algorithms can be used for the remaining compilation steps: data type analysis and implementation, causality analysis, memory allocation, and so on.

The only important remaining question is whether the Lustre-specific compilation algorithms can be combined in optimized compilation flows with the advanced optimization algorithms already implemented in MLIR, which are ultimately responsible for the performance of MLIRgenerated code [5]. To prove this, we have considered a sub-set of the advanced optimization methods of Reference [5] and integrated them as steps of our lus compiler pipeline. Then:

- Using the ML examples (TimeSeries and Resnet50), we show that our compiler already matches (and sometimes outperforms) existing state-of-the-art compilation flows for the reactive language Lustre. 


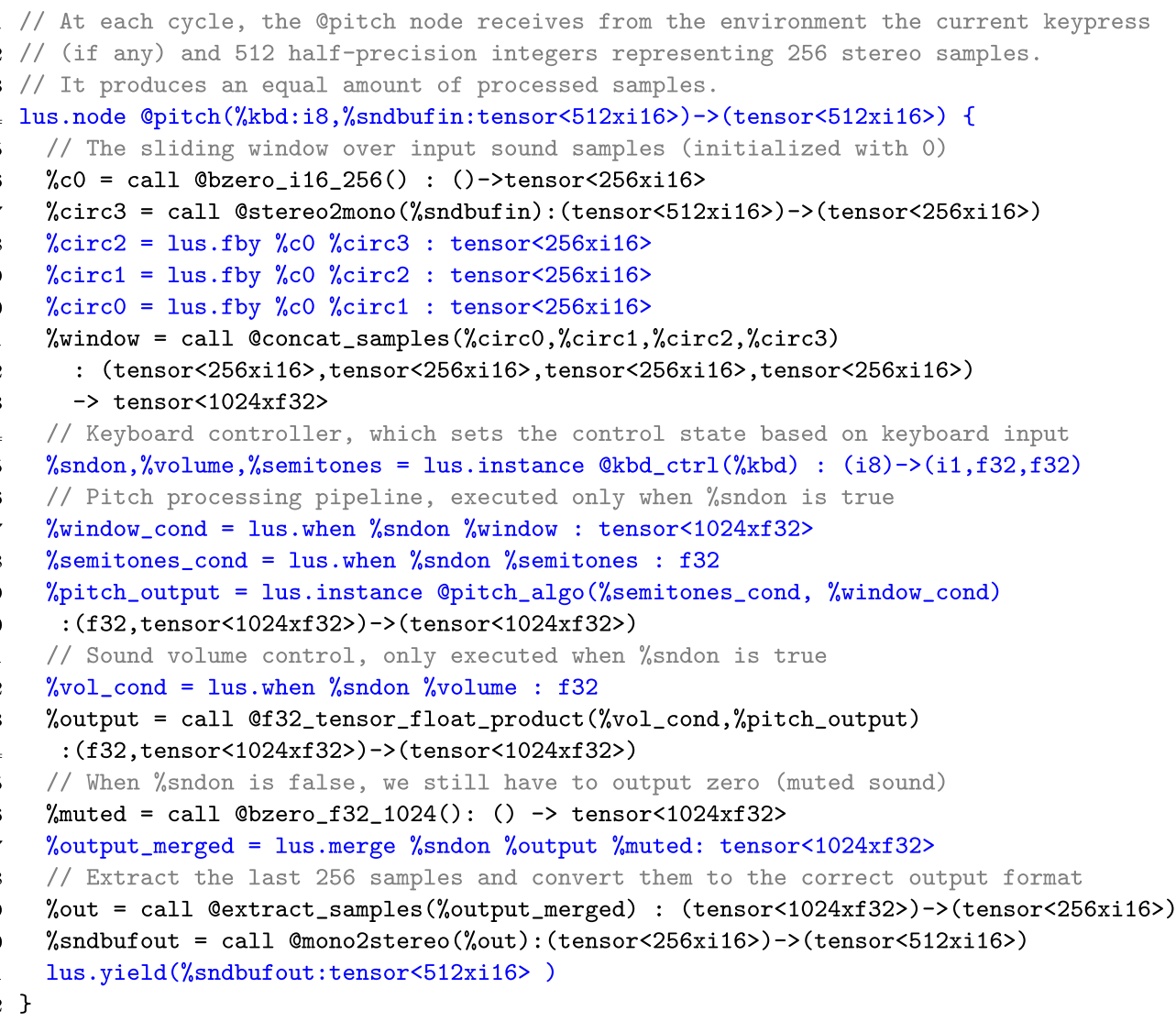

Fig. 14. Pitch control application-top-level node. In blue, lus dialect operations.

- For the vocoder example, we show that its implementation is fast enough to allow real-time execution on low-cost commercial hardware.

The full code of our compiler and of the three use cases is provided at https://github.com/dpotop/ mlir-lus-public. For portability, the implementation of modularity is longjmp-based.

\subsection{The Pitch Tuning Vocoder}

The pitch tuning vocoder cyclically samples the sound input and the other control inputs, updates the control state, performs (if required by the current control status) one step of the pitch tuning algorithm, and drives the sound output.

The pitch processing pipeline, ${ }^{16}$ specified using the abstract aggregate types of MLIR (tensors) and operations of the linalg, scf, and std dialects, is encapsulated in the apitch_algo node.

Figure 14 shows the top-level node apitch of the application. This node concentrates most of the reactive control features: the sliding window, multiple feedback loops, as well as the control

\footnotetext{
${ }^{16}$ Involving Hann filtering to reduce spectral leakage, move to frequency domain using the Fast Fourier transform (FFT), move to polar coordinates to separate magnitude from phase, the additive phase synthesis algorithm that performs the actual pitch change, move back to Cartesian coordinates and then to the time domain using the inverse FFT, and a final step of Hann filtering and additive accumulation.
} 
driven by the user interface, which allows silencing the output (and shutting down unneeded pipeline steps) and altering the pitch correction. The apitch node features all the operations of the lus dialect:

- lus.node and lus.yield to specify the node interface and its output.

- lus.when and lus.merge to enforce conditional execution.

- lus.instance to instantiate nodes @pitch_algo and @kbd_ctrl.

Our compilation flow automatically performs buffer allocation and synthesizes low-level control ensuring the correctness and safety of the implementation. Various MLIR-provided optimizations can be applied in the process, as discussed in Section 5.3.

\subsection{Neural Network Use Cases}

We use the lus dialect to represent the reactive control of two convolutional neural networks: an instance of the $t f$. keras. applications. ResNet50 implementation of the Residual Network [13] and a time series forecasting application ${ }^{17}$ based on LSTM [14].

The more interesting of the two, from a reactive specification standpoint, is the time series application. Indeed, while ResNet is a feedforward network, the LSTM at the core of the time series application is a recurrent layer. Furthermore, the behavior specified by the use case is a multi-rate one: input is cut in sections of five cycles each, with one output produced at the end of each interval. Subsampling is performed on the output of the @lstm node instance. Thus, computations driven by the output of the LSTM are executed at a pace five times slower than the internal computations of the LSTM (grouped in node @lstm). Such multi-rate execution mechanisms are also common in embedded control applications specified in Lustre, and can be used to support multi-period execution [10].

\subsection{Comparison with Lustre}

For all three applications, we have also encoded the same behavior in Lustre, using the open-source state-of-the-art Heptagon compiler. ${ }^{18}$ In doing this, we took advantage of the Heptagon-specific language extensions, which allow for limited array processing using map/fold iterators, to improve performance.

As our use cases only feature fixed-sized data, encoding in Lustre/Heptagon posed no problem. Of course, our Lustre embedding into MLIR is more expressive in this respect, as it can take advantage of the full MLIR type system, which allows for arrays and tensors of dynamic sizes.

But the most interesting part of the comparison concerns the performance figures, summarized in Table 1. To obtain them, the Heptagon-generated code has been compiled with gcc at increasing optimization levels and executed on a Intel Core i5-7600 processor rated at $3.50 \mathrm{GHz}$. The MLIR code has been compiled using two different flows: a default flow without very little MLIR-level optimization, where only common subexpression elimination is performed in addition to the mandatory lowering and normalization passes, and a high-level flow that includes loop nest optimizations representative of compilers for high-performance computing. These loop nest optimizations including loop tiling, packing (copying for cache spatial locality and conflicts) and loop unrolling; they have been implemented in MLIR within the course of a couple of weeks and do not mean to compete with the most agressive, automatically tuned optimizers for tensor computations

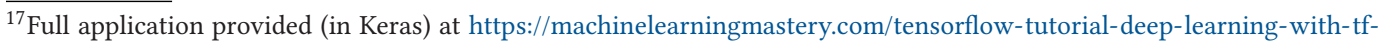
keras/, Section 3.3.

${ }^{18}$ http://heptagon.gforge.inria.fr/.
} 
Table 1. Average Execution Time Per Cycle for the Neural Network Use Cases

\begin{tabular}{|c||r|r|}
\hline Specification \& compilation method & ResNet50 & TimeSeries \\
\hline MLIR/lus & $11.748 \mathrm{~s}$ & $250 \mu \mathrm{s}$ \\
\hline MLIR/lus optimized & $4.873 \mathrm{~s}$ & $130 \mu \mathrm{s}$ \\
\hline Heptagon+gcc-O0 & $151.847 \mathrm{~s}$ & $846 \mu \mathrm{s}$ \\
\hline Heptagon+gcc-O1 & $52.730 \mathrm{~s}$ & $212 \mu \mathrm{s}$ \\
\hline Heptagon+gcc-O2 & $22.592 \mathrm{~s}$ & $148 \mu \mathrm{s}$ \\
\hline Heptagon+gcc-O3 & $5.165 \mathrm{~s}$ & $40 \mu \mathrm{s}$ \\
\hline
\end{tabular}

[5]. For each use case, every six configurations were executed ten times and the smallest figure has been considered in each configuration to reduce the effect of OS interference. ${ }^{19}$

It is no surprise that, in the absence of optimizations, Heptagon-generated code is inefficient. However, on the ResNet50 example, which is representative of large ML applications, the still preliminary and lightweight MLIR loop nest optimizations yield better performance than the stateof-the-art Heptagon+gcc-O3 flow, which leverages the agressive optimizations of the (currently) most performant open-source compiler (gcc).

Given that we only scratched the optimization potential of MLIR [5], these results clearly show that the marriage we propose between MLIR and Lustre has the potential for high runtime performance.

Overall, this evaluation confirms the expressiveness and effectiveness claims, about leveraging existing SSA-based optimizations, the ease of implementing optimizations specific to dataflow synchronous compilers, and the ability to apply loop nest optimizations such as loop tiling to a dataflow synchronous program. This draws a very promising path for the development of specification formalisms, programming languages and compilers for applications where both performance and predictability are important.

\section{RELATED WORK}

We advance the state-of-the-art in SSA by providing the syntactic constructs, structural rules, and semantics extensions allowing the representation of synchronous reactive behavior. This overall objective is fully original.

However, particular aspects of our extension have been covered in previous work, in particular predicated execution $[7,18]$ and the use of semantic absence/undefinedness $[9,15]$. In previous SSA work, predicated execution is explicit: the predicates are represented at all points where they exert control. We allow an implicit predication specification, akin to dataflow semantics, where the absence of inputs determines absence of execution. This requires a clear definition of correctness, which is absent in Reference [7].

Our treatment of semantic absence/undefinedness is also different from that proposed for LLVM [15]. Our objective is not to ensure that non-deterministic code (involving undefined values) preserves its set of traces unchanged under various optimizations. Instead, it is to guarantee that well-defined values are preserved by various optimizations, even in the presence of undefined behaviors affecting other variables. We attain this goal through the abstraction theorem of Section 3.4 .

We also extend previous work on the compilation of synchronous languages [2, 4, 19]. Existing synchronous language compilers profoundly restructure the code to match an execution model (which, as explained in Section 3.1.2, is in most cases sequential function calls, even though multithreaded implementations have also been proposed [16]). We fully avoid this by ensuring that

\footnotetext{
${ }^{19}$ Our experiments do not involve random elements, so we assumed that OS-related interferences are responsible for most of the variance.
} 
every reactive SSA specification can be executed as is, and be subject to any correct SSA transformation. Synchronous-specific code transformations are still possible, but not mandatory.

More important maybe, instead of advancing the compilation of synchronous languages as a separate research field, we show that it can be seen as an extension of classical compilation, allowing the reuse of fundamental techniques for type checking, causality analysis, buffer allocation, and so on.

\section{CONCLUSION}

We presented an embedding of the Lustre synchronous reactive language into SSA, extending the MLIR framework to allow synchronous programming and code generation. We illustrated it by capturing the data processing, computational and reactive control aspects of signal processing and ML applications. Our MLIR extension remains fully compatible with SSA-based analysis and transformations while also capturing the synchronous composition of concurrent state machines, logical and physical time synchronization, and compilation passes specific to synchronous languages, such as clock calculus, causality analysis, initialization analysis, bounded-memory, and clock-directed code generation.

While there had long been connections between the semantics of functional languages, SSA and dataflow synchronous languages, this article describes the concrete extension of SSA capturing the necessary elements for reactive control system. Furthermore, retaining all SSA-based compilation algorithms and reusing existing code unaware of the the specific synchronous concurrency semantics allows a tighter integration of reactive system modeling frameworks with the computationally intensive and general-purpose capabilities of MLIR- and LLVM-based frameworks for HPC and ML.

Our work paves the way for unified design and implementation flows where the specification of reactive control, state machines, and real-time ressource orchestration become first class citizens on par with parallelization and optimization in general-purpose frameworks. One key objective here is to lift our unification between reactive control and data parallelism from the level of the MLIR intermediate format (not meant for direct programming) to that of high-level specification languages such as Lustre, Keras, or Jax. Since synchronous concurrency is highly popular in safety-critical environments, one important future direction is to fully formalize the core MLIR components our extension relies upon, and to prove its correctness. A related direction consists in exploring syntax and refinements for state-machines and control automata expressed in the Esterel synchronous language as well as hardware design languages such as Bluespec and Chisel. It is also important to further investigate which SSA-based algorithms can benefit the compilation flow of reactive control systems and how domain-specific methods for synchronous languages can enable greater automation and guarantees in the composition and memory management of concurrent systems in general.

\section{REFERENCES}

[1] ARINC. 2010. ARINC 653: Avionics Application Software Standard Interface. Part 1-Required Services. Revision 3.

[2] A. Benveniste, P. Caspi, S. A. Edwards, N. Halbwachs, P. LeGuernic, and R. de Simone. 2003. The synchronous languages 12 years later. Proc. IEEE 91, 1 (Jan. 2003).

[3] J. L. Bergerand, P. Caspi, D. Pilaud, N. Halbwachs, and E. Pilaud. 1985. Outline of a real time data flow language. In Proceedings of the RTSS.

[4] D. Biernacki, C. J.-L. Cola, G. Hamon, and M. Pouzet. 2008. Clock-directed modular code generation for synchronous data-flow languages. In Proceedings of the LCTES.

[5] Uday Bondhugula. 2020. High Performance Code Generation in MLIR: An Early Case Study with GEMM. Retrieved from https://arXiv:2003.00532. 
[6] T. Bourke, L. Brun, P.-E. Dagand, X. Leroy, M. Pouzet, and L. Rieg. 2017. A formally verified compiler for lustre. In Proceedings of the PLDI.

[7] L. Carter, B. Simon, B. Calder, L. Carter, and J. Ferrante. 1999. Predicated static single assignment. In Proceedings of the PACT.

[8] R. Cytron, J. Ferrante, B. K. Rosen., M. N. Wegman, and F. K. Zadeck. 1991. Efficiently computing static single assignment form and the control dependence graph. ACM Trans. Program. Lang. Syst. 13, 4 (1991).

[9] D. Demange and D. Pichardie Y. Fernández de Retana. 2018. Semantic reasoning about the sea of nodes. In Proceedings of the CC.

[10] K. Didier, D. Potop-Butucaru, G. Iooss, A. Cohen, J. Souyris, P. Baufreton, and A. Graillat. 2019. Correct-byConstruction parallelization of hard real-time avionics applications on off-the-shelf predictable hardware. ACM Trans. Archit. Code Optim. 16, 3 (2019), 24:1-24:27.

[11] L. Gérard, A. Guatto, C. Pasteur, and M. Pouzet. 2012. A modular memory optimization for synchronous data-flow languages: Application to arrays in a Lustre compiler. In Proceedings of the LCTES.

[12] N. Halbwachs. 2005. A synchronous language at work: The story of Lustre. In Proceedings of the Memocode.

[13] K. He, X. Zhang, S. Ren, and J. Sun. 2016. Deep residual learning for image recognition. In Proceedings of the CVPR.

[14] S. Hochreiter and Jürgen Schmidhuber. 1997. Long short-term memory. Neural Comput. 9, 8 (1997), 1735-1780.

[15] Juneyoung Lee, Yoonseung Kim, Youngju Song, Chung-Kil Hur, S. Das, D. Majnemer, J. Regehr, and N. P. Lopes. 2017. Taming undefined behavior in LLVM. In Proceedings of the PLDI.

[16] X. Li and R. von Hanxleden. 2012. Multithreaded reactive programming-The Kiel Esterel processor. IEEE Trans. Comput. 61, 3 (2012).

[17] MLIR. 2020. Multi-level Intermediate Representation Compiler Framework (MLIR). Retrieved from https://mlir.llvm. org/.

[18] K. J. Ottenstein, R. A. Ballance, and A. B. MacCabe. 1990. The program dependence web: A representation supporting control-, data-, and demand-driven interpretation of imperative languages. SIGPLAN Not. 25, 6 (June 1990), $257-271$.

[19] D. Potop-Butucaru, S. A. Edwards, and G. Berry. 2007. Compiling Esterel. Springer.

[20] B. K. Rosen, M. N. Wegman, and F. K. Zadeck. 1988. Global value numbers and redundant computations. In Proceedings of the POPL.

[21] SSA. 2020. Static Single Assignment Book (in progress). Retrieved from http://ssabook.gforge.inria.fr/latest/book.pdf.

[22] The TensorFlow Authors. 2017. Implementation of Control Flow in TensorFlow. Retrievd on 1 Oct., 2021 from http: //download.tensorflow.org/paper/white_paper_tf_control_flow_implementation_2017_11_1.pdf.

Received August 2021; revised November 2021; accepted December 2021 